\title{
Three-dimensional computed tomography for a mediastinal basal pulmonary artery
}

Running Head: Mediastinal basal pulmonary artery

Keitaro Matsumoto, MD, Naoya Yamasaki, MDª Tomoshi Tsuchiya, MDª Takuro Miyazaki, MDa, Koichi Tomoshige, $\mathrm{MD}^{\mathrm{a}}$, Hideyuki Hayashi, $\mathrm{MD}^{\mathrm{b}}$, Kazuto Ashizawa, $\mathrm{MD}^{\mathrm{c}}$, and Takeshi Nagayasu, $\mathrm{MD}^{\mathrm{a}}$

${ }^{a}$ Division of Surgical Oncology, Department of Translational Medical Sciences, Nagasaki University Graduate School of Biomedical Sciences, Nagasaki, Japan

${ }^{\mathrm{b}}$ Division of Radiological Science, Department of Radiology and Radiation Biology, Nagasaki University Graduate School of Biomedical Sciences, Nagasaki, Japan.

${ }^{c}$ Clinical Oncology Center, Nagasaki University Hospital, Nagasaki, Japan

Address correspondence to:

Keitaro Matsumoto

Division of Surgical Oncology, Department of Translational Medical Sciences, Nagasaki University Graduate School of Biomedical Sciences, 1-7-1 Sakamoto, Nagasaki 852-8501, Japan

Phone: +81-95-819-7304

Fax: +81-95-819-7306

E-mail address: kmatsumo@ nagasaki-u.ac.jp

Key Words: Anatomy, Pulmonary arteries/veins, Imaging, Thoracoscopy/VATS, Lung cancer surgery 


\begin{abstract}
Anatomical variations of the pulmonary artery increase the risks for vessel injury and critical mistakes during pulmonary artery resection. A case that underwent left lower lobectomy for lung cancer with a mediastinal lingular and basal pulmonary artery that had been detected preoperatively by three-dimensional computed tomography is presented. During thoracoscopic surgery, the mediastinal basal pulmonary arteries were found with careful dissection. The pulmonary arteries were divided, and left lower lobectomy was performed safely. This rare variation of the left pulmonary artery is important to detect before lobectomy to ensure safe surgery. In such cases, three-dimensional computed tomography is necessary.
\end{abstract}


There is some variation of the branching pattern of the pulmonary artery, particularly in the left lung. The most common variation is the mediastinal lingular pulmonary artery (1). Understanding of pulmonary artery abnormalities is most important for lung resection under video-assisted thoracic surgery (VATS) because of the difficulties associated with the limited field of view with VATS. A case in which a mediastinal lingular pulmonary artery was found preoperatively by three-dimensional computed tomography (3D-CT) is reported. This variation is extremely rare and very critical for left lobectomy, particularly for left upper lobectomy. In this case, left lower lobectomy was performed safely because the variation was discovered preoperatively. Preoperative 3D-CT is very important to identify pulmonary artery variations.

\section{Case Report}

A 78-year-old man was referred to our department with suspected lung cancer in the left lower lobe. Enhanced CT revealed a 23-mm-diameter tumor in the lower lobe that was strongly suspected to be lung cancer. We decided to perform left lower lobectomy for lung cancer, and 3D images of the pulmonary artery and vein were constructed by 3D-CT preoperatively, which is done routinely to confirm the branching of vessels to ensure that the operation is performed safely and quickly. 3D-CT revealed that two branches were flowing into the lower lobe. One was the normal branch through the back of the left main bronchus, which was relatively narrower than usual. Another was the so-called mediastinal lingular artery, which went through the front of the left main bronchus and entered the lower lobe (S8+9b), providing arteries into the lingular segment. The tumor was diagnosed as lung cancer by fine needle aspiration cytology, and lower lobectomy was performed. First, the main pulmonary artery and mediastinal lingular pulmonary artery were carefully dissected from the back or the front of the hilum, and after confirmation of the pulmonary arteries flowing into the lower lobe, they were divided by endostaplers. Then, the lower pulmonary vein and lower bronchus were divided by endostaplers, and the left lower lobe was removed from the chest cavity. Subsequently, mediastinal lymph node and hilar lymph node dissections were performed by taping the pulmonary arteries. The main pulmonary artery was 
narrower than usual, but because of the preoperative 3D-CT examination, it was relatively easy and safe to identify these arteries. The tumor was diagnosed as adenocarcinoma, with a pathological stage of T1bN0M0. The patient's postoperative course was stable, and he was discharged 15 days after the operation.

\section{Comment}

In the lung surgery field, pulmonary vascular variations are not uncommon. Sobotich et al. reported that $16.4 \%$ of patients had pulmonary vessel variations, and $47.8 \%$ of these variations occurred in the left lung (1). Among the variations of the left pulmonary artery, the most common variation is the so-called mediastinal lingular artery, which is the first branch of the left main pulmonary artery. The mediastinal lingular artery, which includes the A4+A5 type and either the A4 or A5 type, has been reported in 27.3\% of cases (2). However, a mediastinal basal artery is extremely rare, and only a few cases have been reported $(3,4)$. There might be cases that are not noticed during operations because it is difficult to identify if it is not detected preoperatively. The present case is the first report of a combination mediastinal lingular and basal artery with left main pulmonary artery, and the first time it has been shown clearly by 3D-CT. In such cases, if there is no information about pulmonary artery abnormalities, the lobectomy would be difficult to perform safely, and sometimes mistakes could be made (4), particularly when incomplete fissures are present. Incomplete oblique fissures in the left lung have been reported to occur in from $21 \%$ to $46.6 \%$ of cases $(5,6)$. This means that care is needed in many cases of left lobectomy.

Complete VATS has been accepted as a standard operation for stage IA lung cancer $(7,8)$. This is a very useful method to see the details of the structure while performing the operation. On the other hand, it is relatively difficult to see the whole lung and the sizes of arteries or veins. This disadvantage could cause misunderstanding of vessel abnormalities during surgery. 3D-CT could overcome this disadvantage because it clearly shows the structure of the pulmonary vessels and bronchi preoperatively. Of course, it is possible to detect these abnormalities by axial views of chest CT, but it takes a long time to learn and to perform a careful evaluation before operation. 3D-CT could provide easy and reliable images of 
abnormalities of pulmonary vessels and bronchi for many surgeons. Thus, 3D-CT is very useful for lung surgery and should be performed before surgery if possible.

A mediastinal lingular and basal artery is very rare, but it is very important to identify to facilitate safe left lobectomy. 3D-CT provides strong support for confirming pulmonary vessel structure. 


\section{References:}

1. Subotich D, Mandarich D, Milisavljevich M, Filipovich B, Nikolich V. Variations of pulmonary vessels: some practical implications for lung resections. Clin Anat 2009;22:698-705.

2. Yamashita H. Variations in the pulmonary segments and the bronchiovascular trees. In: Roentgenologic Anatomy of the lung. Tokyo: Igaku-shoin; 1978:70-107.

3. Kataoka K, Nishikawa T, Fujiwara T, Matsuura M. A case of lung cancer with an extremely rare branching pattern of the left A5+8+9+10 pulmonary artery. Jpn J Lung Cancer 2010;50:362-365.

4. Moriyama S, Miyoshi K, Tada A, Kurosaki T. A case report of abnormal branching of left A8+9 pulmonary artery. Jpn J Chest Surg 2009;23:58-61.

5. Lukose R, Paul S, Sunitha DM et al. Morphology of the lungs: Variations in the lobes and fissures. Biomedicine 1999;19:227-32.

6. Meenakshi S, Manjunath KY, Balasubramanyam V. Morphological variations of the lung fissures and lobes. Indian J Chest Dis Allied Sci 2004;46:179-82.

7. Flores RM, Ihekweazu U, Dycoco J et al. Video-assisted thoracoscopic surgery (VATS) lobectomy: catastrophic intraoperative complications. J Thorac Cardiovasc Surg 2011;142:1412-7.

8. Swanson SJ, Herndon JE 2nd, D'Amico TA et al. Video-assisted thoracic surgery lobectomy: report of CALGB 39802--a prospective, multi-institution feasibility study. J Clin Oncol 2007;25:4993-7. 


\section{Figure Legends:}

Figure 1. Three-dimensional CT shows the left main pulmonary artery (open arrow) and the left mediastinal lingular and basal pulmonary artery (arrow). Their size looks similar, and the left main pulmonary artery includes A4, A6, A9a, and A10, and the left mediastinal pulmonary artery includes A5, A8, and A9b.

Figure 2. Picture taken during the operation (left) and its schema (right). The mediastinal pulmonary artery comes from the back of the superior pulmonary vein and enters the basal segment after providing A5. 
Figure1

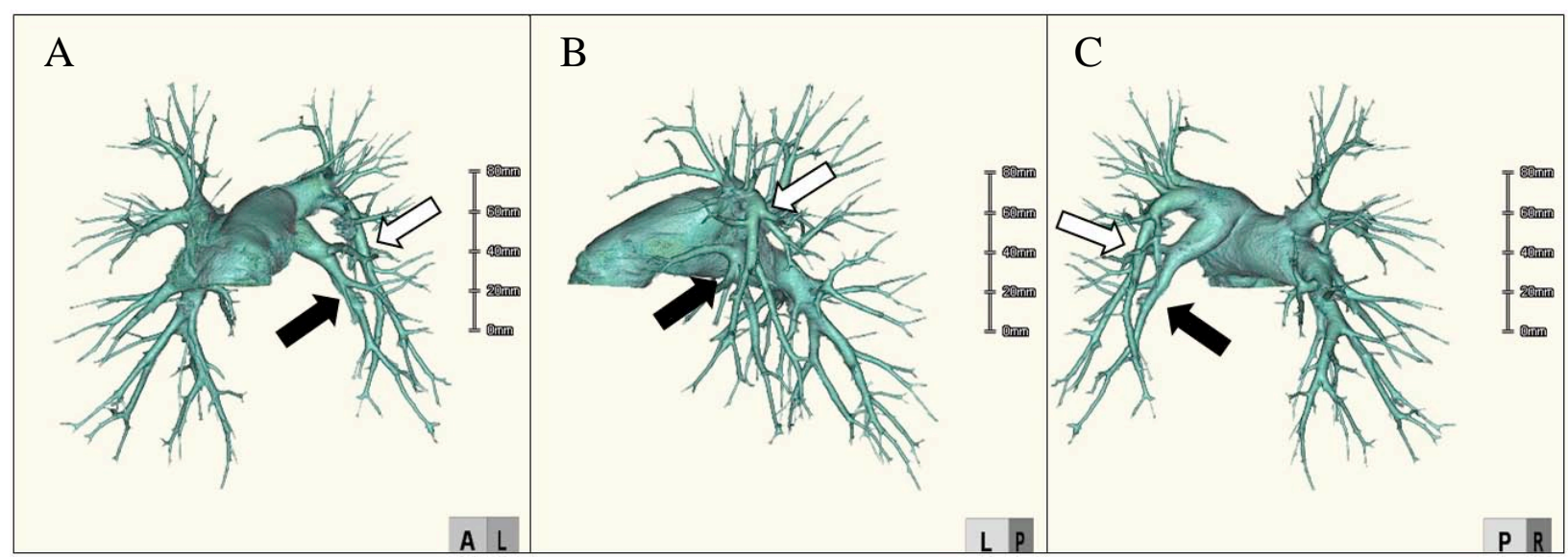


Figure2
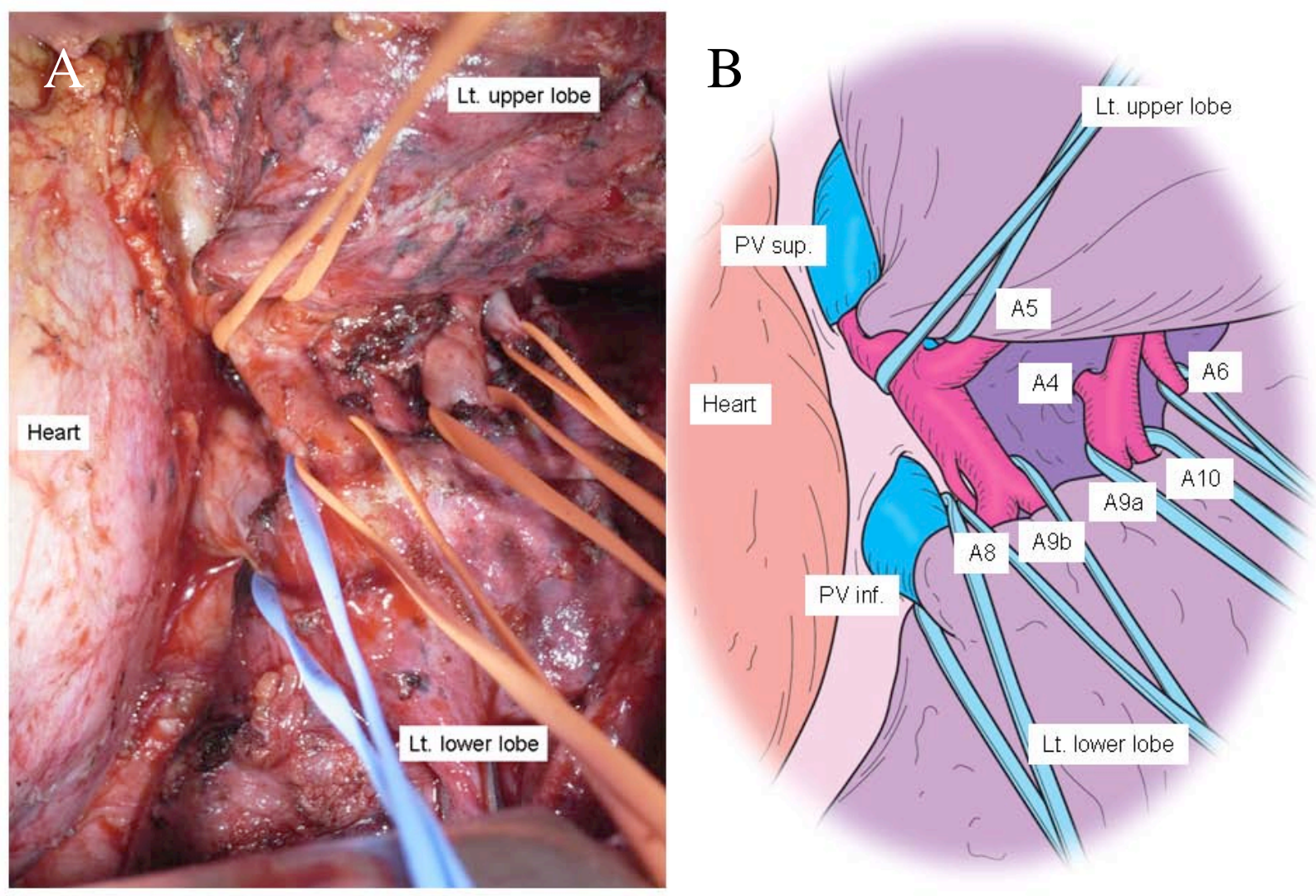